\title{
A Representação da Desigualdade Econômica em Crianças e Adolescentes de Nível Socioeconômico Diferentes
}

\author{
The Representation of Economic Inequality in Children and \\ Adolescents of Different Social Economical Status \\ Antonio Roazzi ${ }^{\star}$, Maria da Graça B. B. Dias, Maira Roazzi \\ Universidade Federal de Pernambuco, Recife, Brasil
}

\begin{abstract}
Resumo
Existe uma controvérsia na literatura relativa ao desenvolvimento da representação da desigualdade econômica. A questão levantada por estas investigações é: Os atributos do conhecimento desta noção de desigualdade econômica são coletivos ou individuais? Uma investigação foi planejada com uma amostra de 85 participantes de origem sócio-econômica diferenciada: 30 adolescentes oriundos de famílias de NSE médio e 55 adolescentes oriundos de famílias de NSE baixo (30 morando com os pais e 25 morando na rua). Foram utilizados desenhos de pessoas representando ocupações que os participantes deviam ordenar em função de remuneração, atribuir salário e avaliar a satisfação. Os resultados apontam para a existência de uma relação entre pertencer a um determinado grupo sócio-cultural e aspectos cognitivos da forma de representação das desigualdades econômicas em nossa sociedade. De fato, as recompensas de salários associados com ocupações em diferentes posições na estratificação, são representadas diferentemente em função do nível sócio-econômico e experiência sociocultural do participante. Assim, é possível considerar que a estrutura do sistema de atividades sobre-individuais na qual a criança está inserida desempenha um papel crucial no desenvolvimento de formas específicas de representação da desigualdade social. Estes resultados e suas implicações para a compreensão do processo de socialização são discutidos.
\end{abstract}

Palavras-Chave: Desigualdade econômica; psicologia econômica; representações sociais; socialização.

\begin{abstract}
There is a controversy in the literature concerning the development of children's representations of economic inequality. The issue is whether the attributes of children's concept of economic inequality are fundamentally collective or individual? An investigation was planned with a sample of 85 subject of different social economical status (SES): 30 adolescents from high SES families and 55 from low SES families (30 living with their parents and 25 living in the street). Pictures of people representing different professions were used, which participants had to put in order according to remuneration, attributing wages and evaluating satisfaction. The results point out the existence of a relationship between belonging to a certain social-cultural group and cognitive aspects of how economic inequalities are represented in our society. In fact, remunerations associated with different types of occupations were represented differently depending of social economical status and cultural experience of the participant. Thus, it's possible to consider that the structure of the system of super-individual activities in which the child is inserted plays a crucial part in the development of specific forms of social inequality representation. These results and its implications for an understanding of the socialization process are discussed. Keywords: Economic inequality; economic psychology; social representations; socialization.
\end{abstract}

A forma como crianças e adolescentes compreendem noções econômicas é até hoje pouco conhecido, apesar de um redespertado interesse acadêmico e não acadêmico nesta área, sobretudo pelas crescentes desigualdades socioeconômicas das duas últimas décadas decorrentes de uma sociedade cada vez mais globalizada ${ }^{1}$ (Danziger \& Gottschalk, 1995; Duncan

\footnotetext{
* Endereço para correspondência: Rua Francisco da Cunha, 654/801, Ed. Villa das Pedras, Bairro Boa Viagem, 51020-041, Recife, PE. Fones: (00 55 81) 2126.8272 e 2126.7330 (Universidade); Fax: (00 55 81) 2126.7331. E-mail dos autores: roazzi@gmail.com,mgbbdias@gmail.com

${ }^{1}$ No Brasil estas desigualdades são cada vez maiores. Como é bem conhecido, principalmente, a partir da ampla divulgação dos resultados da pesquisa empírica de Barros, Henriques e Mendonça (2000), algo em torno de 1/3 dos brasileiros são pobres, muito embora o país seja relativamente rico quando sua renda per capita é colocada em perspectiva internacional e tenha passado por fases relativamente recentes de crescimento acelerado.
}

\& Brooks-Gunn, 1997; Duncan, Brooks-Gunn \& Klebanov, 1994; Glyn \& Miliband, 1994; Jones, 1995; Kerstenetzky , 2002; Sklar, 1995; Wilkinson, 1994a, 1994b, 1996). De fato, ainda há poucos estudos que podem ser encontrados na literatura abordando este fenômeno, além disso, muitos aspectos destas noções nos últimos anos não foram abordados adequadamente do ponto de vista evolutivo. Destas noções econômicas, uma se refere ao desenvolvimento da representação da desigualdade econômica, (e.g., Burgar, Cheyne, \& Jahoda, 1989; Chafel, 1996; Dickinson, 1990; Emler, \& Dickinson, 1985; Furby, 1979; Jahoda, 1983; Leahy, 1981, 1983, 1990; Leiser, 1983; Leiser, Sevón, \& Levy, 1990; Weinger, 2000). A respeito desta noção existem na literatura pontos de convergências e também de divergências. Por exemplo, análises preliminares das evidências da literatura apon- 
tam para a existência de uma relação entre, por um lado, pertencer a um determinado grupo sociocultural e por outro, os aspectos cognitivos da forma de representação das desigualdades econômicas em nossa sociedade (Emler \& Dickinson, 1985; Nunes \& Roazzi, 1999; Roazzi, Sales \& Nunes, 1996). Isto é, existem evidências que a estrutura do sistema de atividades sobre-individuais, na qual a criança está inserida, desempenha um papel crucial no desenvolvimento de formas específicas de representação da estratificação social e da desigualdade social. Entretanto, existem também na literatura evidências apresentando resultados contrastantes (Burgard, Cheyne \& Jahoda, 1989).

Emler e Dickinson (1985) realizaram um estudo no qual solicitava-se a uma amostra de crianças escocesas entre 7 e 12 anos de idade, de diferentes NSE, para estimar a renda semanal de várias ocupações. Foram encontradas diferenças significativas em função do NSE, mas não em função da idade. A estimativa média das crianças de NSE médio por cada ocupação era mais alta do que as crianças de NSE baixo. Além do mais, as crianças de NSE médio diferenciaram muito mais os ganhos entre as várias profissões do que as crianças de NSE baixo. As crianças de NSE médio apresentaram também um nível mais sofisticado de justificativas sobre a desigualdade, produzindo também um número mais variado de razões.

Quando comparada com a variável idade não apareceu diferença na estimativa de renda das ocupações dada pelas crianças, enquanto que em termos de NSE essa diferença surgiu de forma marcante. Isso aponta para uma compreensão mais rápida da complexidade do sistema social das crianças de NSE médio. Em uma investigação similar Dickinson (1990) observou em uma amostra de jovens escoceses que tanto jovens de nível NSE baixo como de NSE médio eram capazes de ordenar diferentes profissões de acordo com a remuneração e aceitavam este sistema baseado nas diferenças como justo e natural. Além do mais, os jovens de NSE médio defendiam com maior veemência esta desigualdade e a justificavam mais frequentemente em função de uma maior escolarização e "expertise". Com estes resultados, Emler e Dickinson (1985) concluíram que as representações sociais das desigualdades econômicas são mais amplas e salientes na classe média e, portanto, as crianças de NSE médio teriam mais oportunidades para assimilar o conhecimento compartilhado no seio de sua classe social, como também as crenças sobre as desigualdades de renda.

Este estudo foi replicado por Burgard et al. (1989) com uma amostra de crianças alemãs entre 8 e 12 anos. Foram encontradas, ao contrário do estudo de Emler e Dickinson (1985), diferenças significativas com a idade, mas não com a classe social. A observação destas divergências nos levou a realizar uma outra réplica do estudo de Emler e Dickinson com crianças brasileiras de NSE diferentes (Roazzi et al., 1996). Neste contexto, no Brasil, onde as diferenças entre classes sociais são mais acentuadas do que na sociedade européia, deveria ser mais fácil detectar o papel desempenhado pelo nível socioeconômico no desenvolvimento do conceito de desigualdades. Assim, as questões que nós colocamos foram: Os atributos do conhecimento desta noção de desigualdade econômica são coletivos ou individuais? Quais as diferenças na representação da desigualdade econômica em termo de remuneração? Nesta investigação (Roazzi et al., 1996) solicitou-se às crianças ( $\mathrm{N}=180$, idades entre 7 e 12 anos) de diferentes NSE (baixo e médio) que estimassem os salários (pela distribuição de 80 fichinhas) e o nível educacional de 24 ocupações, metade das crianças de NSE baixo estavam frequientando a escola e metade tinha deixado a escola. As ocupações incluíam algumas que dependiam de formação universitária (e.g., médico), ocupações de alto status que não exigem formação universitária, mas onde a educação é, de fato, necessária (e.g., gerente de banco, político), ocupações de colarinho branco de status inferior onde as exigências educacionais são indefinidas (e.g., policial), qualificações vocacionais (e.g., motorista de ônibus) e nenhuma qualificação exigida (e.g., gari). Os resultados confirmaram o que tinha sido encontrado por Emler e Dickinson (1985) ao ser observada uma interação significativa entre NSE e as estimações das crianças dos níveis de remuneração das diferentes profissões. As diferenças dos níveis de remuneração, entre as diferentes profissões, foram muito mais marcadas nas crianças de NSE médio do que nas crianças de NSE baixo. Nenhuma interação idade versus ocupação foi encontrada como no estudo de Burgard et al. (1989).

A partir destas considerações uma outra investigação foi planejada com uma amostra de participantes de origem socioeconômica diferenciada. O objetivo foi explorar como, em crianças e adolescentes, se desenvolve a representação das desigualdades sócio-econômicas e como este nível de representação interage com o meio social de origem das crianças. Qual a imagem que crianças e adolescentes possuem das várias atividades profissionais exercidas por vários grupos de pessoas em nossa sociedade e como estas estão relacionadas com o conceito de remuneração. Basicamente este estudo é similar ao de Roazzi et al. (1996). No presente estudo ampliou-se a faixa etária da amostra (8 a 19 anos de idade) e incluiu-se um outro grupo, os dos Meninos de rua.

Este interesse surgiu da necessidade de não somente focalizar os aspectos mais específicos da representação da desigualdade econômica - assunto pouco estudado. Mas, sobretudo, de entender o significado que uma noção tão importante como esta assume para a compreensão, por parte da criança/adolescente, do mundo em que vive, compreensão esta, mediada pelo tipo de processo de socialização ao qual está submetida (Nunes \& Roazzi, 1999).

\section{Método}

\section{Participantes}

A amostra do estudo era composta por 30 crianças/adolescentes oriundos de famílias de NSE médio e 55 adolescentes oriundos de famílias de NSE baixo (30 morando com os pais e 25 morando na rua). Os três grupos que serão denominados respectivamente: Médio, Baixo e Rua (ou Meninos de rua), apresentam a seguinte distribuição de meninos e meninas: $16-14$ (Médio), 15 - 15 (Baixo), e $15-13$ (Rua). O critério adotado para a definição da classe social para o grupo Médio e Baixo foi o tipo de escola (pública ou particular) freqüentada. A adoção deste critério "tipo de escola freqüentada" como indicador do nível sócio-econômico decorre de várias pesquisas realizadas no Recife (ver, por exemplo, Carraher, \& Schliemann, 1982; Roazzi, 1988) nas quais observou-se uma relação muito estreita entre a variável escola freqüentada e 
nível sócio-econômico da família de origem. O grupo de meninos de rua, diferentemente dos outros dois grupos, não freqüentavam a escola, e se encontravam vivendo na rua há pelo menos dois anos. Foram consideradas as informações sobre o tipo de profissão dos pais como também o nível de educação dos mesmos como informação de controle complementar. A média de idade, desvio padrão e número de participantes para cada grupo de acordo com a faixa etária foram respectivamente: Médio (8-12 anos 11.5 -. 85 - 10; 13-15 anos $14.0-.92-8 ; 16-19$ anos $17.8-1.33-12)$, Baixo (8-12 anos $10.6-1.6$ - 9; 13-15 anos 14.1 - .81 - 16; 16-19 anos $16.4-.55-5)$, e Rua (8-12 anos $11.5-.71-10 ; 13-15$ anos $14.1-.83-8 ; 16-19$ anos $16.4-.53-7)$.

\section{Material}

1. Doze desenhos de figuras humanas (desenhados em cartolinas $14 \times 12 \mathrm{~cm}$.) representando de maneira estilizada várias ocupações profissionais. Abaixo de cada desenho encontrava-se escrita a ocupação da figura representada. As ocupações foram as seguintes: (a) Dono de cadeia de supermercados (em seguida chamaremos Empresário), (b) Deputado estadual, (c) Juiz, (d) Médico, (e) Delegado de polícia, (f) Assistente Social, (g) Mestre de obra, (h) Motorista de ônibus, (i) Camelô, (j) Vigia de prédio, (k) Empregada doméstica e (l) Gari;

2. Desenho seriado de cinco rostos apresentando diferentes níveis de satisfação obtidos através da curvatura da boca;

3. Notas de dinheiro de vários valores (simulando notas reais) e conjuntos de notas com a inscrição indicando valer um salário mínimo (SM).

\section{Procedimento}

Em uma primeira fase, eram apresentados a cada participante desenhos de pessoas que representam as doze ocupações acima descritas. Estas gravuras tinham o propósito de representar uma série de profissões diferentes em termos de rendimentos reais. Elas foram escolhidas a partir de um estudo piloto visando averiguar o conhecimento das mesmas nos três grupos de participantes. Antes de começar a entrevista o entrevistador levantava várias questões sobre as ocupações para que os participantes pudessem se familiarizar com as mesmas. Em seguida era solicitado aos participantes ordenar as 12 ocupações ou profissões de acordo com suas estimativas sobre sua real remuneração. O participante devia ordenar tais ocupações de acordo com conceitos de remuneração de um extremo (remuneração mais baixa $=1$ ) para outro extremo (remuneração mais alta $=12$ ).

Em seguida o entrevistador separava quatro ocupações, Empresário, Médico, Motorista e Gari e solicitava que o participante distribuísse dinheiro de brinquedo que pensavam ser pago realmente a cada deles no final do mês. O participante devia também indicar o nível de satisfação de cada profissional em uma escala Likert de 7 pontos formada por sete torres ordenadas de diferentes alturas que vão de altamente insatisfeita (torre mais baixa) para altamente satisfeita (torre mais alta). Em seguida os participantes eram novamente encorajados a distribuir o dinheiro de acordo com o que elas achavam certo cada classe profissional ganhar.

\section{Resultados}

\section{Análise do Nível de Remuneração e de Satisfação}

As médias das estimativas de renda mensal das quatro ocupações em número de salários mínimos indicam que os participantes de NSE médio eram capazes de diferenciar mais as ocupações de alto status das com baixo status do que os outros dois grupos de participantes. Nesta tendência a maior discriminação é mais clara para a questão "Acha" ("Quanto você acha que ganha”) do que para a questão "Deveria" ("Quanto você acha que deveria ganhar"). Nesta última questão a distribuição era muito mais igualitária entre as várias ocupações para os três grupos. Com o objetivo de verificar estas observações os dados foram tratados estatisticamente através de uma análise de variância do tipo 3 (Grupo: Médio, Baixo e Meninos de Rua) x 3 (Idade: 8-12, 13-15, 16-19) x 4 (Ocupação: Empresário, Médico, Motorista, Gari) tendo esta última variável como o fator intra-sujeitos e a remuneração (número de SM atribuídos a cada ocupação) como variável dependente. Esta análise foi realizada separadamente para a distribuição da remuneração à questão "Quanto você acha que ganha?" E para a questão "Quanto você acha que deveria ganhar??. Esta análise produziu efeitos principais significativos em Ocupação tanto para a questão "Acha" $[\underline{F}(3,180)=16.98 ; p<.001]$ como para a questão "Deveria" $[\underline{F}(3,195)=9.05 ; p<.001]$. É interessante também registrar o efeito principal marginalmente significativo da variável Grupo na questão "Acha" $[\underline{F}(2,60)$ $=3.09 ; p<.053]$. Efeito interativo significativo foi encontrado entre Grupo e Ocupação $[\underline{F}(6,180)=2.45 ; p<.027]$ somente para a questão "Acha” (Figura 1).

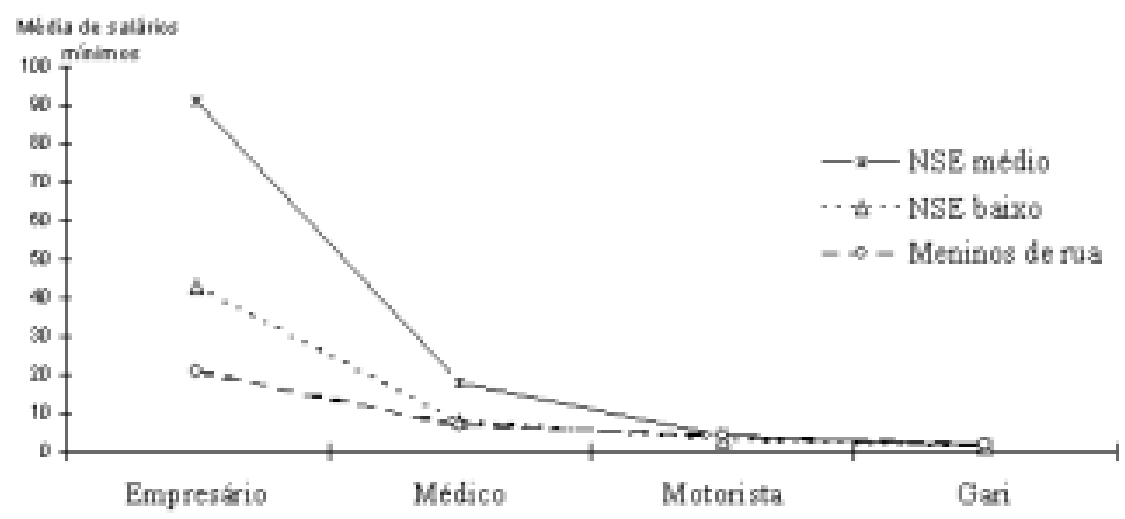

Figura 1. Interação NSE x Ocupação. VD: “Quanto você acha que ganha?”. 
Análises a posteriori das diferenças entre as médias das quatro ocupações através do Teste a posteriori de Tukey revelaram que, tanto para a questão " $A$ cha" quanto para a questão "Deveria", todas as comparações entre as quatro ocupações foram significativas $(\not<.01)$ - a exceção da comparação Motorista-Gari na questão "Deveria”. Os níveis de remuneração de cada ocupação entre os três grupos para a questão "Acha" foram também comparados através de teste a posteriori indicando que o grupo NSE médio apresentou remunerações mais altas do que o grupo NSE baixo e Meninos de rua tanto para a ocupação Empresário $(p<.01)$ quanto para a ocupação Médico $(p<.05)$. Foi constatada também uma remuneração média mais alta do NSE baixo em relação aos Meninos de rua $(p<.05)$ somente para Empresário. Comparações entre as quatro ocupações para cada grupo indicaram que foi atribuído ao Empresário um nível de remuneração mais alto em relação às outras três profissões tanto no grupo NSE baixo $(p<.05)$ como no grupo NSE médio $(p<.01)$. Nenhuma diferença significativa foi observada no grupo Meninos de rua.

Em relação aos julgamentos sobre o nível de satisfação observa-se que o grupo de NSE médio era capaz de diferenciar mais as ocupações de alto status das com baixo status do que os outros dois grupos. Esta tendência podia ser observada nas três faixas etárias. Além do mais, a discriminação era mais clara, sobretudo, para a questão "Acha" do que para a questão "Deveria". Nesta última questão o julgamento do nível de satisfação era muito mais igualitário entre as várias ocupações para os três grupos. Com o objetivo de verificar estas observações os dados foram tratados estatisticamente através de uma análise de variância do tipo 3 (Grupo: Médio, Baixo e Meninos de Rua) x 3 (Idade: 8-12, 13-15, 16-19) x 4 (Ocupação: Empresário, Médico, Motorista, Gari) tendo esta última variável como o fator intra-sujeitos e nível de satisfação como variável dependente. Esta análise era realizada separadamente para a distribuição da remuneração à questão "Acha" e para a questão "Deveria". Foram encontrados efeitos principais significativos para a variável Grupo tanto para a questão "Acha" $[\underline{F}(2,76)=3.88 ; p<.025]$ quanto para a questão "Deveria" $[\underline{F}(2,74)=5.31 ; p<.007]$. O mesmo ocorreu para Ocupação também tanto para a questão " $A$ cha"
$[\underline{F}(3,228)=84.08 ; \not<<.001]$ quanto para a questão "Deveria" $[\underline{F}(3,222)=3.90 ; p<.010]$. Efeito interativo significativo foi encontrado entre Grupo e Ocupação $[\underline{F}(6,288)=$ $4.52 ; p<.001]$ somente para a questão "Acha" (Figura 2).

Análises a posteriori das diferenças entre as médias dos três grupos através do Teste a posteriori de Tukey indicaram que os Meninos de rua apresentam níveis de satisfação mais altos do que os participantes de NSE baixo (tanto na questão "Acha" $p<.05$ quanto na questão "Deveria" $p<.01)$ e os de NSE médio (tanto na questão " $A$ cha" $p<.01$ quanto na questão "Deveria" $p<.05)$. Comparações entre as quatro ocupações através do teste a posteriori de Tukey revelaram que, para a questão " $A c h a$ " todas as comparações entre as quatro ocupações eram significativas $(p<.01)$. Para a questão "Deveria" a ocupação de médico foi julgada como mais satisfeita do que a ocupação de Empresário $(p<.01)$ e Motorista $(p<.05)$.

Os níveis de satisfação de cada ocupação, entre os três grupos para a questão "Acha" foram também comparados através de teste a posteriori. Os resultados indicaram que o grupo Meninos de rua apresentou remunerações mais altas do que o grupo NSE baixo e NSE médio tanto para a ocupação de Motorista $(p<.01)$ quanto para a ocupação de Médico ( $p<.05$ Rua vs. Baixo; $p<.01$ Rua vs. Médio).

Comparações entre as quatro ocupações para os grupos de NSE médio e baixo indicaram haver diferenças significativas $(p<.01)$ entre todas as ocupações. Para os Meninos de rua as diferenças estatisticamente significativas $(p<.05)$ foram observadas somente entre Gari e as outras três ocupações.

\section{Razão das Estimativas de Remuneração e de Satisfação}

Uma outra maneira de análisar as estimativas produzidas pelos participantes é em termos de razão entre a ocupação que recebe a mais alta remuneração (Empresário) e a ocupação que recebe a remuneração mais baixa (Gari). $\mathrm{Na}$ Figura 3 estão descritas as médias da razão da estimativa de remuneração entre as ocupações Empresário/Gari de acordo com a variável Grupo. Antes de tudo é possível observar, na questão "Acha", diferenças muito mais marcantes entre as profissões Empresário/Gari nos participantes de NSE médio, do que nos outros dois grupos, especialmente o grupo de Meninos de rua. Na questão "Deveria" as diferenças são bem menores.

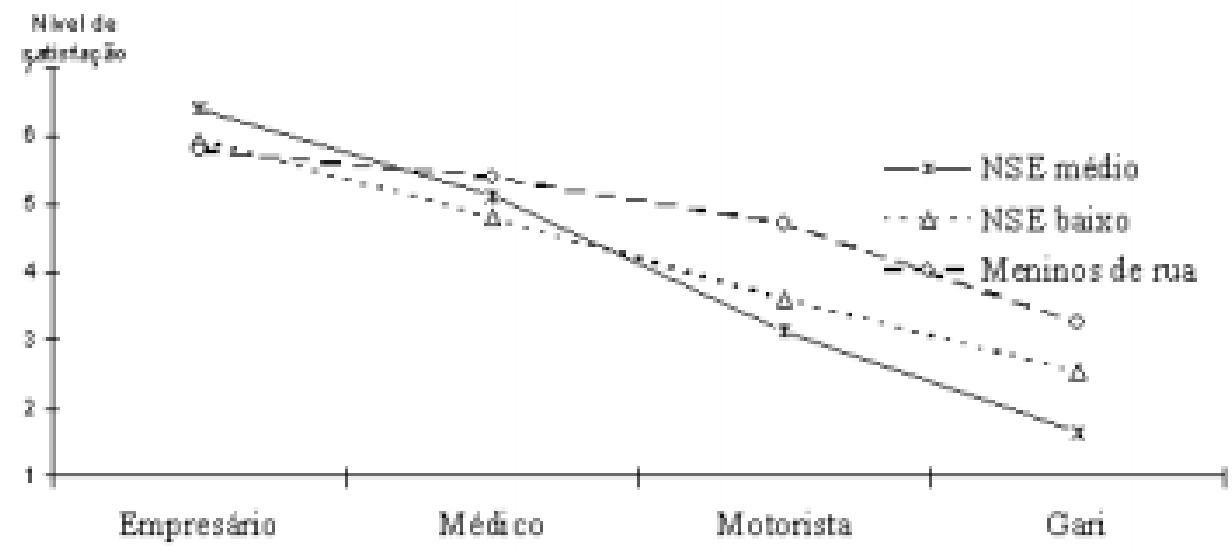

Figura 2. Interação NSE x Ocupação.VD: Nível de satisfação na questão "Acha". 


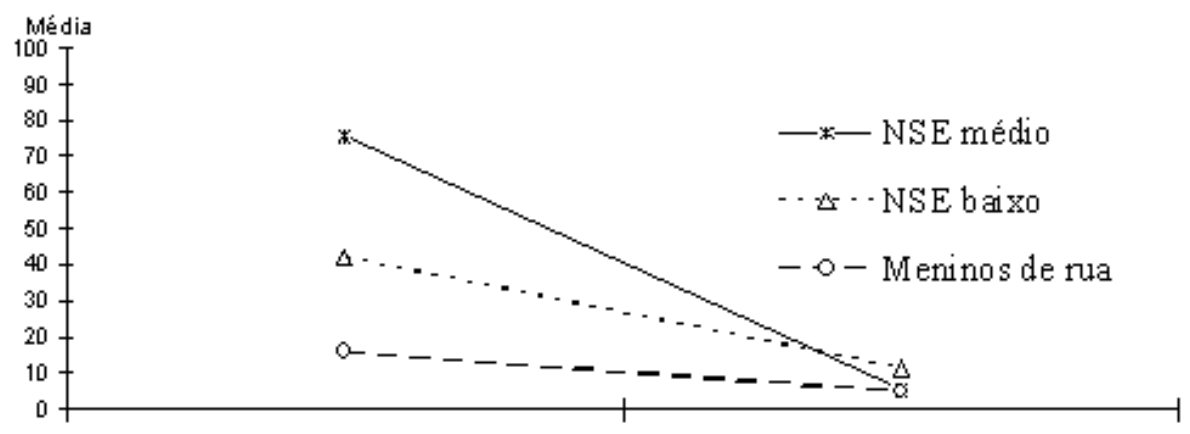

Quanto você acha que ganha? Quanto você acha que deveria ganhar?

Figura 3. Interação Grupo x Questão Acha/Deveria.VD: Razão da remuneração entre Empresário e Gari nas duas condições "acha que ganha" e "acha que deveria ganhar".

Estes dados foram tratados estatisticamente através de uma análise de variância envolvendo fator "entre" Grupo (3), como fator "intra" a Questão Acha/Deveria (2) e razão entre a remuneração das ocupações Empresário/Gari como variável dependente. A análise produziu um efeito principal significativo para Questão $[F(1,69)=14.99 ; p<.001]$ como efeito interativo Grupo x Questão $[F(2,69)=3.21$; $p<.046]$. As médias das razões para os três grupos foram comparadas através do Teste de Tukey. Esta análise mostrou uma diferença significativa da média das razões entre as duas questões em todos os três grupos ( $p<.01)$. Quando os três grupos foram comparados em relação à questão " $A$ cha" o grupo de NSE médio apresentou médias mais altas do que os outros dois grupos $(p<.01)$. A diferença entre NSE baixo e Meninos de rua foi também significativa $(p<.05)$. Pelo contrário, quando os três grupos foram comparados em relação à questão "Deveria" não foram observadas diferenças significativas.

Na Figura 4 estão apresentadas as médias da razão da estimativa do nível de satisfação entre as ocupações Empresário/Gari de acordo com a variável Grupo. Observa-se na questão "Acha", diferenças muito mais marcantes entre as profissões Empresário/Gari nos participantes de NSE médio do que nos outros dois grupos. Na questão "Deveria" as diferenças são bem menores.
Estes dados foram tratados estatisticamente através de uma análise de variância envolvendo fator "entre" Grupo (3), como fator "intra” a Questão Acha/Deveria (2) e razão entre a estimativa de satisfação das ocupações Empresário/ Gari como variável dependente. A análise produziu um efeito principal significativo para Grupo $[F(2,83)=9.44$; $p<.001]$ e para Questão $[F(1,83)=81.4 ; p<.001]$, como efeito interativo Grupo x Questão $[F(2,83)=3.37$; $p<.039]$. As médias das razões dos três grupos foram comparadas através do Teste de Tukey mostrando que o grupo de NSE médio apresentou razões mais altas do que os outros dois grupos $(p<.01)$. Nenhuma diferença foi observada entre NSE baixo e Meninos de rua. Em seguida foram analisadas as diferenças do efeito interativo. Enquanto na questão "Deveria", nenhuma diferença foi observada entre os três grupos, na questão "Acha" o grupo de NSE médio apresentou razões mais altas do que os outros dois grupos $(p<.01)$. As comparações entre as duas questões para cada grupo mostraram haver razões mais altas na questão " $A$ cha" do que na questão "Deveria" nos três grupos $(p<.01)$.

\section{Análise da Ordenação das Ocupações}

As médias das ordenações das ocupações em função do nível de remuneração que os participantes achavam em

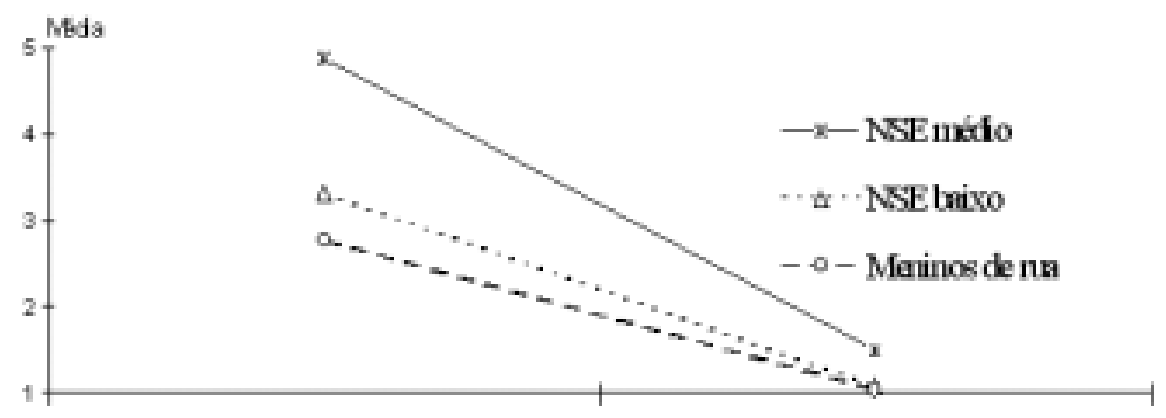

Quanto você acha que ganha? Quanto você acha que deveria ganhar?

Figura 4. Interação Grupo x Questão Acha/Deveria. VD: Razão das estimativas do nível de satisfação entre Empresário e Gari nas duas condições "acha que ganha" e "acha que deveria ganhar". 
nossa sociedade encontram-se apresentadas na Tabela 1. Observa-se claramente que os participantes de NSE médio apresentam médias mais altas nas ocupações de alto status social e/ou que precisam um nível universitário - Empresário, Deputado, Juiz e Médico. Única exceção é observada no grupo de 16-19 anos onde a média de Empresário é idêntica nos três grupos e a média de Médico é mais alta para os participantes de NSE baixo. Nas ocupações de Delegado e Assistente Social o padrão é misto. Para as restantes profissões de baixo status social, que em geral não requerem uma qualificação específica, as médias do grupo de NSE médio não são mais altas do que nas dos outros dois grupos - Mestre de obra, Motorista, Camelô, Vigia, Empregada Doméstica e Gari. As diferenças se acentuam entre os três grupos especialmente para a profissão de Gari. Assim, existe uma tendência no grupo NSE médio, de operar uma maior diferenciação das remunerações das várias ocupações de acordo com o status social e o tipo de qualificação envolvida.

As diferenças entre os vários grupos de participantes no tipo de ordenação das ocupações de acordo com o nível de remuneração foram analisadas através de análises de variâncias do tipo 2 (Grupo: Médio, Baixo e Rua) x 3 (Idade: 8-12, 13-15, 16-19) por cada ocupação, tendo a ordenação destas como variável dependente. Diferenças entre participantes em função da variável Grupo foram encontradas para a ocupação de Deputado $[F(2,64)=4.31$; $p<.010]$, Camelô $[F(2,64)=3.96 ; p<.024]$ e Gari $[F$ $(2,64)=2.83 ; p<.044]$. Em relação à variável Idade, como também a interação Grupo x Idade não foi observado nenhum efeito significativo. Em seguida foi realizada uma análise a posteriori das diferenças entre as médias (Tukey) entre os três grupos por cada ocupação (ver Tabela 1) que mostraram haver diferenças significativas. Na profissão de Deputado, o grupo Meninos de Rua apresentou julgamentos mais baixos em relação ao grupo de NSE médio $(p<.01)$ e ao grupo NSE baixo $(p<.05)$. Na ocupação $\mathrm{Ca}-$ melô, o grupo NSE baixo apresentou julgamentos mais baixos comparado ao grupo de NSE médio $(p<.05)$ e ao grupo Meninos de Rua ( $p<.01)$. Enfim, na ocupação Gari, é o grupo de NSE médio que apresenta julgamentos mais baixos em relação ao grupo de NSE baixo $(p<.05)$ e ao grupo Meninos de Rua $(p<.05)$.

Os resultados das análises acima apontam para um efeito interativo entre Grupo e Ocupação visto que os participantes de NSE médio, em relação aos outros dois grupos, tendem a diferenciar mais entre o nível de remuneração, entre ocupação de alto status social e que envolvem cursos universitários e entre ocupação de status social menos elevado e que requer qualificações menos especializadas. Para verificar esta interação, as diferenças entre os três grupos no tipo de ordenação das ocupações de acordo com o nível de remuneração, foram analisadas através de uma análise de variância do tipo 3 (Grupo: Médio, Baixo e Rua) x 3 (Idade: 8-12, 13-15, 16-19) x 12 (Ocupações: Empresário, Deputado, Juiz, Médico, Delegado, Assistente Social, Mestre de Obra, Motorista, Camelô, Vigia, Empregada Doméstica e Gari; fator “intra”) tendo a ordenação das ocupações como variável dependente. A análise mostrou como significativos o efeito principal Ocupação $[F(11,704)=$ $214.55 ; p<.001]$ e o efeito interativo Grupo x Ocupação [ $F$ $(22,704)=1.58 ; p<.046$; ver Figura 5]. Este efeito interativo da variável Ocupação com Grupo e não com Idade confirma nossa observação sobre a existência de uma forma diferenciada de julgar a distribuição das remunerações em nossa sociedade nos três grupos comparados. No grupo de NSE médio esta distribuição é mais diferenciada do que nos outros dois grupos - NSE baixo e Meninos de Rua.

De modo geral, estes resultados apontam na mesma direção dos dados relativos às atribuições de remuneração. Isto é, uma discriminação mais clara das diferenças existentes em nossa sociedade, em termos de remuneração entre as várias ocupações profissionais por parte dos participantes de NSE médio em relação aos outros dois grupos - sobretudo os Meninos de rua.

Para verificar, de maneira mais detalhada e qualitativa,

Tabela 1

Médias das Ordenações (e Anova) das Ocupações em Função do Nível de Remuneração na Condição "Acha que Ganha" de Acordo com Idade e Grupo

\begin{tabular}{|c|c|c|c|c|c|c|c|c|c|c|c|c|c|c|c|}
\hline \multirow{2}{*}{ Ocupações } & \multicolumn{3}{|c|}{$8-12$ anos } & \multicolumn{3}{|c|}{ 13-15 anos } & \multicolumn{3}{|c|}{ 16-19 anos } & \multicolumn{3}{|c|}{ Anova: $F$} & \multicolumn{3}{|c|}{ Teste de Tukey } \\
\hline & $\mathrm{Me}$ & Bai & Rua & $\mathrm{Me}$ & Bai & Rua & $\mathrm{Me}$ & Bai & Rua & $\mathrm{NSE}$ & Idade & $\mathrm{NxI}$ & $\mathrm{MxB}$ & $\mathrm{MxR}$ & $\mathrm{BxR}$ \\
\hline Empresário & 11.2 & 10.4 & 11.1 & 11.2 & 10.9 & 11.0 & 11.5 & 11.5 & 11.5 & 1.20 & 1.07 & .30 & & & \\
\hline Deputado & 10.6 & 10.0 & 8.37 & 10.9 & 10.7 & 10.4 & 10.6 & 9.75 & 9.50 & $4.31^{\mathrm{b}}$ & 2.04 & .65 & & *⿻丷木 & * \\
\hline Juiz & 10.3 & 9.50 & 9.25 & 10.6 & 9.85 & 10.6 & 10.8 & 10.5 & 9.75 & 1.85 & 1.02 & .31 & & & \\
\hline Médico & 9.50 & 8.75 & 8.37 & 8.75 & 8.86 & 7.80 & 8.83 & 9.25 & 8.25 & 1.71 & .38 & .32 & & & \\
\hline Delegado & 7.60 & 10.2 & 8.37 & 8.12 & 8.28 & 8.80 & 8.08 & 7.50 & 8.00 & 2.09 & .91 & 2.77 & & & \\
\hline Assist. Social & 7.50 & 7.25 & 6.75 & 7.37 & 7.57 & 6.20 & 7.16 & 7.75 & 7.25 & 1.28 & .02 & .35 & & & \\
\hline Mest. de Obra & 4.80 & 4.75 & 6.62 & 4.50 & 5.35 & 5.00 & 4.16 & 6.25 & 4.00 & 1.79 & .48 & 1.39 & & & \\
\hline Motorista & 4.80 & 5.00 & 4.75 & 5.25 & 5.36 & 5.20 & 5.58 & 5.25 & 5.75 & .03 & 1.17 & .09 & & & \\
\hline Camelô & 3.10 & 1.50 & 3.12 & 2.50 & 2.43 & 3.20 & 3.42 & 2.25 & 4.00 & $3.96^{\mathrm{a}}$ & .09 & .63 & * & & *⿻丷木 \\
\hline Vigia & 3.60 & 4.50 & 4.37 & 3.25 & 4.29 & 4.20 & 3.50 & 3.50 & 3.25 & 2.14 & .76 & .44 & & & \\
\hline Emp. Domes. & 2.80 & 3.25 & 3.75 & 4.00 & 2.78 & 4.00 & 2.41 & 2.50 & 3.00 & 1.44 & 1.99 & .76 & & & \\
\hline Gari & 2.20 & 2.87 & 3.12 & 1.50 & 1.93 & 1.60 & 1.92 & 2.00 & 3.75 & $2.83^{\mathrm{a}}$ & 2.15 & .57 & & * & * \\
\hline
\end{tabular}

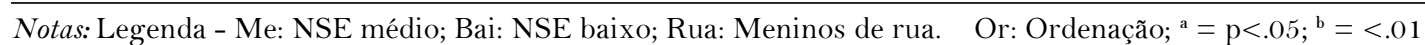




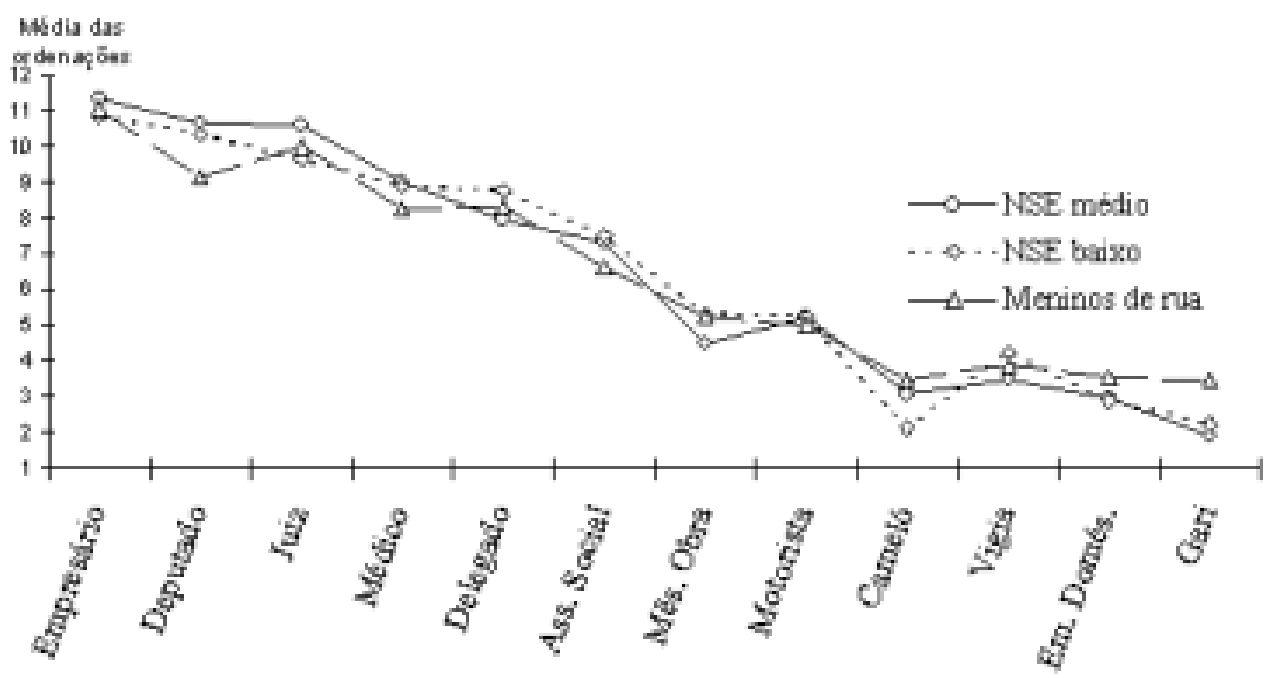

Figura 5. Interação NSE x Ocupação. VD: Ordenação das ocupações em função da remuneração.

estas diferenças entre os três grupos de adolescentes, em seguida foram comparados através de uma Similarity Structure Analysis (SSA - Análise da Estrutura de Similaridade; ver Borg, \& Groenen, 2005; Roazzi, 1995) os perfis relativos às ordenações das ocupações em função da remuneração dos participantes de cada grupo com os perfis dos participantes de um dos outros dois grupos perfazendo um total de três comparações (NSE médio vs. NSE baixo; NSE médio vs. Meninos de rua; e NSE baixo e Meninos de rua). Não foram comparados os três grupos conjuntamente devido ao excessivo número de variáveis, sendo comparadas multidimensionalmente alcançando níveis de "stress" altos e, portanto não confiáveis.

O importante desta análise multidimensional é a possibilidade de averiguarmos a estrutura relacional entre os grupos. Os resultados da análise paramétrica nos indicam que os adolescentes de NSE médio possuem uma compreensão mais clara das diferenças que caracterizam as diferentes ocupações profissionais em termos de remuneração. Esta compreensão do grupo de NSE médio se respalda em um dado de realidade concreto, e não de desejo ou desconhecimento. Hipotetizamos, antes de tudo, que, do ponto de vista espacial, os perfis deste grupo (o perfil de cada participante é representado por um ponto) estejam mais próximos entre si do que os pontos de um dos outros dois grupos comparados. Visto que a compreensão das diferenças remunerativas reflete uma mesma realidade, de qualquer maneira compartilhada em algum aspecto, hipotetizamos, também, que a estrutura relacional entre os dois grupos seja do tipo modular, refletindo uma relação ordenada entre os dois grupos comparados. De fato, por quanto um grupo não compartilhe de maneira parecida com um outro grupo as diferenças remunerativas que realmente caracterizam nossa sociedade, os aspectos parcialmente compartilhados sempre refletem aspectos da mesma realidade, existindo sempre uma relação. A terceira hipótese procede destas duas primeiras. Na região central da estrutura modular sempre iremos encontrar o grupo de participante que compartilha, de forma mais parecida com a realidade, as diferenças que caracterizam os níveis de remuneração em nossa sociedade. Assim sendo, na comparação NSE médio e NSE baixo ou Meninos de rua, os perfis do primeiro grupo se reagruparão na região central, enquanto que os perfis do segundo ou terceiro grupo se espalharão em volta do primeiro de forma concêntrica. De alguma maneira, esperamos observar o mesmo ao comparar o grupo de NSE baixo e Meninos de rua, visto que este último grupo discrimina as diferenças entre as ocupações em termos de remuneração de forma menos parecida aos grupos de NSE médio e NSE baixo.

Nas Figuras 6, 7 e 8 (próxima página) são apresentadas as projeções ou mapas resultantes das três análises SSA comparando os três grupos. Na Figura 6 comparam-se os grupos de NSE médio e NSE baixo. A distribuição dos perfis mostra haver uma estrutura modular com os perfis do grupo de NSE médio no centro e os perfis de NSE baixo distribuído em volta da região ocupada espacialmente pelo primeiro grupo. Entretanto, observam-se seis participantes de NSE baixo na região central dos participantes de NSE médio, mostrando haver alguns participantes de NSE baixo compartilhando uma compreensão parecida com os participantes de NSE médio da distribuição do nível de remuneração. Só um participante de NSE médio encontrase fora da região central ocupada pelos seus pares.

Esta estrutura modular é encontrada também na comparação dos grupos de NSE médio e Meninos de rua (Figura 7). Os perfis dos Meninos de rua, assim como o dos participantes de NSE baixo (Figura 6) encontram-se mais espalhados do que os perfis de NSE médio, indicando maior variação nas ordenações das profissões. Na Figura 8, onde são comparados os grupos de Meninos de rua e de NSE baixo, são estes últimos que se encontram reagrupados em uma região central com Meninos de rua distribuídos em volta desta região. Somente dois participantes Meninos de rua podem ser observados misturados na região central dos participantes 
b

b

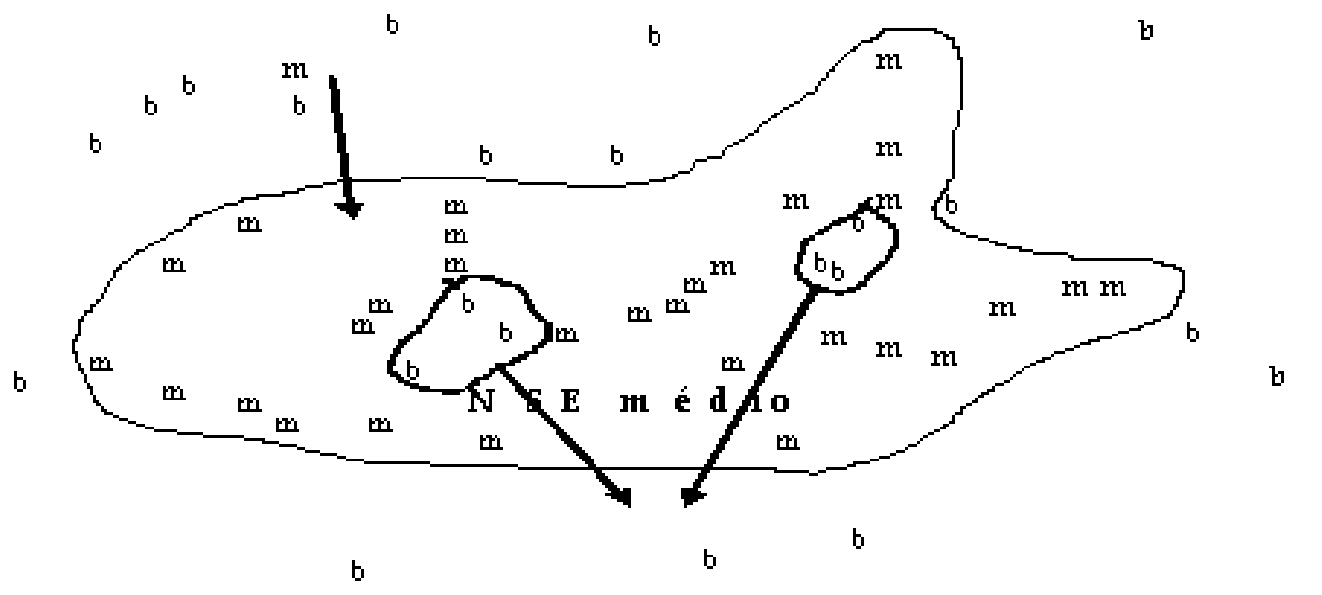

b

N S E b a i $\mathbf{~ o ~}$

Coordenada 1 versuscoordenada 2 dasoluço tridimensional. Stress 20227

Figura 6. Análise SSA comparativa entre os grupos NSE baixo e NSE médio a partir dos perfis relativos às ordenações das ocupações em função da remuneração.

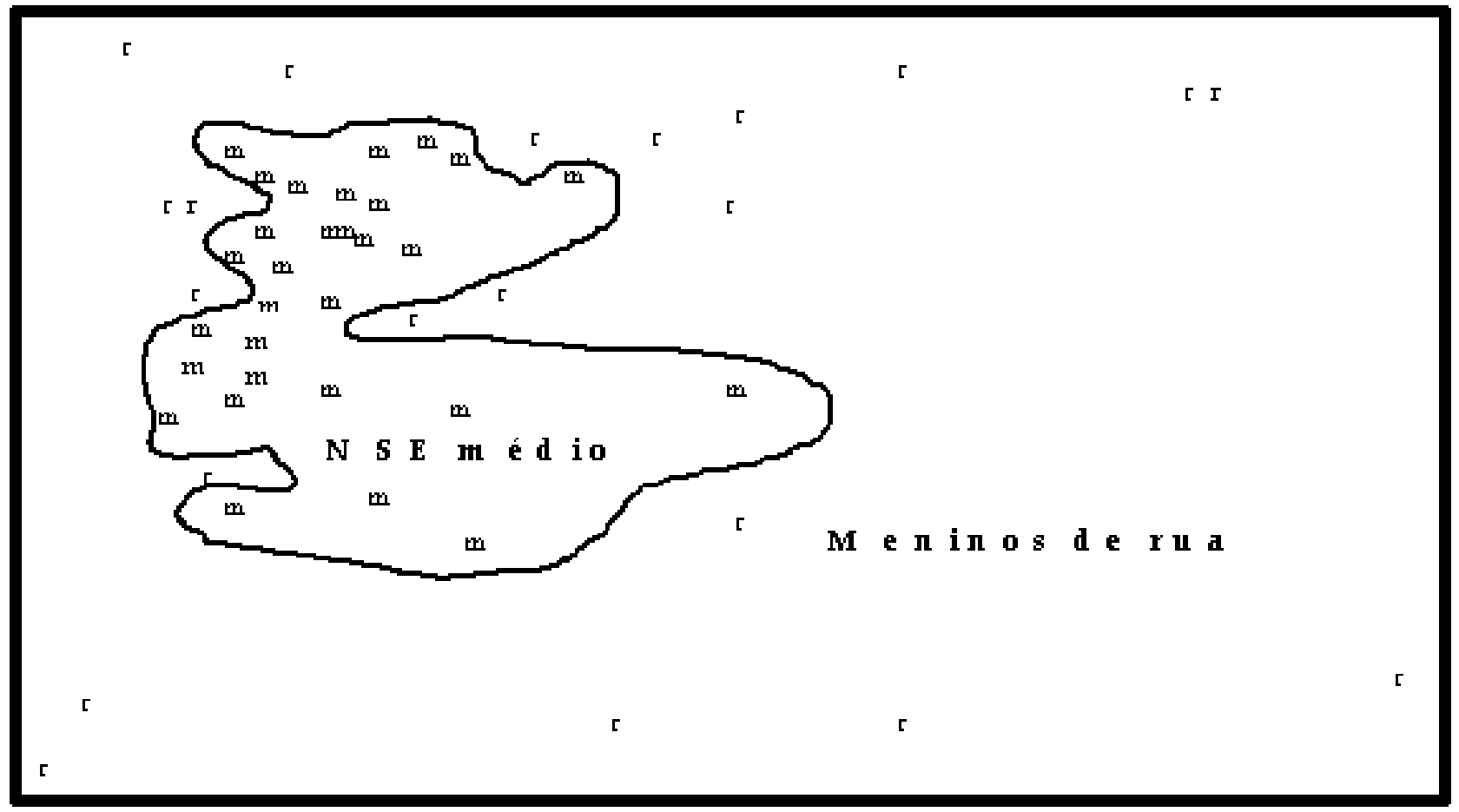

Coordenzda 1 versuscoordenzda 2 dasoluğo tridimensional. stress.17485

Figura 7. Análise SSA comparativa entre os grupos NSE médio e Meninos de rua a partir dos perfis relativos às ordenações das ocupações em função da remuneração. 


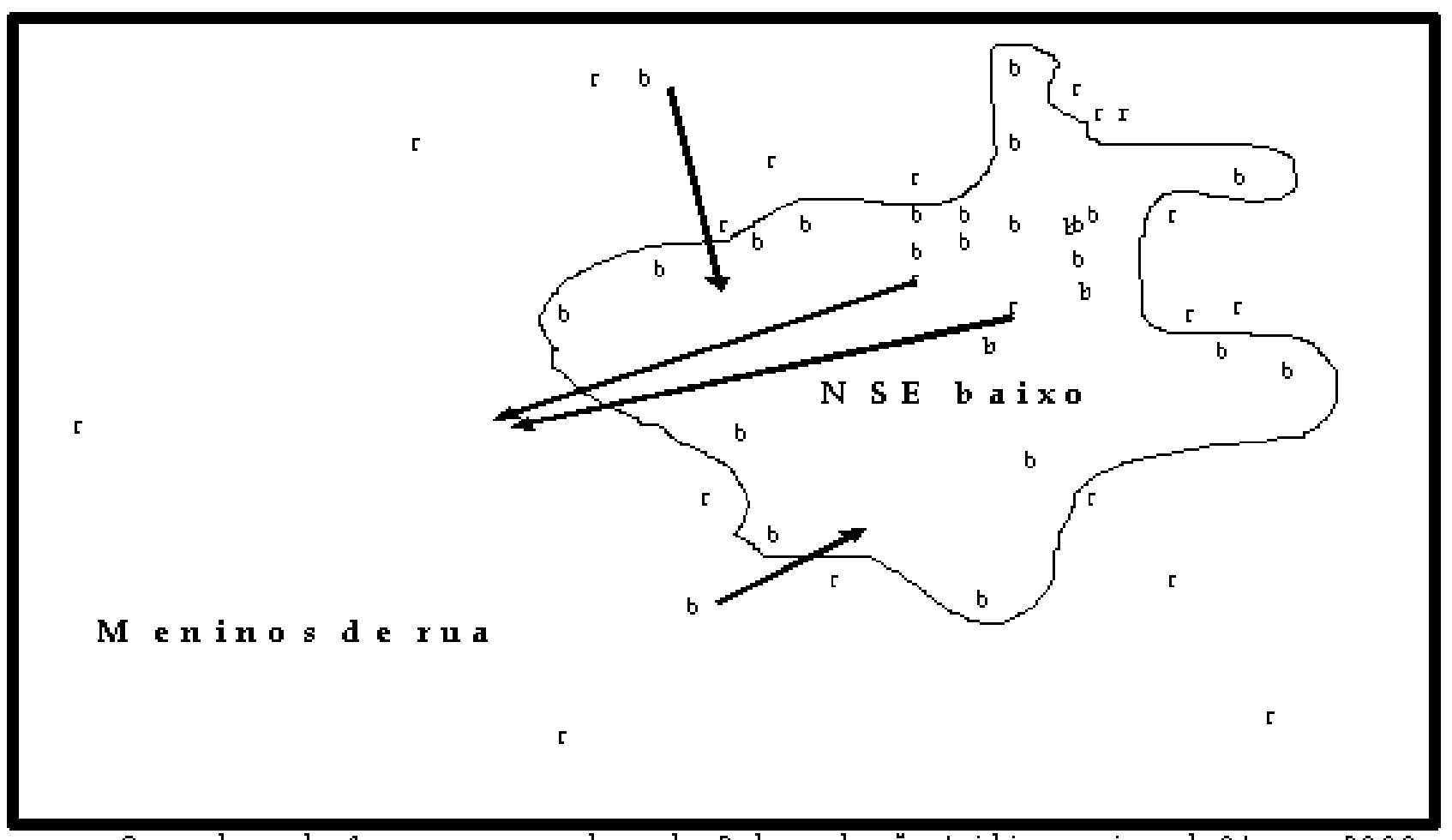

Coordenada 1 versuscoordenada 2 dasoluğotridimensional. Stress.20s

Figura 8. Análise SSA comparativa entre os grupos NSE baixo e Meninos de rua a partir dos perfis relativos às ordenações das ocupações em função da remuneração.

de NSE baixo; ao mesmo tempo apenas participantes de NSE baixo encontra-se fora desta região central.

\section{Discussão}

O objetivo principal desta investigação foi obter uma melhor compreensão da representação de estratificação social e da desigualdade econômica em crianças e adolescentes de NSE diferentes, focalizando mais especificamente a relação entre ocupações e seus respectivos níveis de remuneração. Os resultados desta investigação apontam que participantes de NSE médio, NSE baixo e Meninos de rua constroem mundos consensuais diferentes no que diz respeito às representações relativas a estratificação social, status social e nível de remuneração entre as profissões. Isto é, as recompensas de salários associados com ocupações em diferentes posições na estratificação, são representados diferentemente em função do nível sócio-econômico e experiência sociocultural do participante. Respaldam-se assim os resultados dos estudos realizados por Emler e Dickinson (1985) na Escócia e Roazzi et al. (1996) no Brasil, mas não a investigação de Burgard et al. (1989) na Alemanha - onde foi observada somente uma interação na remuneração das ocupações versus idade (e não NSE).

Em uma investigação mais recente de tipo qualitativo, Weinger (2000) usando técnicas projetivas, explorou a percepção das classes sociais e as escolhas de amizades correspondentes, em uma amostra de crianças (5-14 anos) norteamericanas de NSE médio e baixo. As análises das entrevistas mostraram claramente a existência, desde as faixas etárias menores, de inúmeros preconceitos sobre como riqueza e pobreza eram vistas, e que estes pré-julgamentos afetavam a maneira como era percebido o estabelecimento de possíveis relações de amizade. Estes resultados são um alerta para o fato de que desde pequena as crianças internalizam as divisões que caracterizam nossa sociedade, causadas pelos grandes desníveis de renda, e que estas internalizações são capazes de minar a possibilidade de desenvolver relações de amizade e compreensão mútua entre as pessoas.

Portanto, estes resultados corroboram todas aquelas investigações sobre a compreensão das crianças e adolescentes da estratificação social em diferentes países, mostrando que crianças e adolescentes possuem consciência de diferenças nos salários e status social entre as profissões. Explicam estas diferenças atribuindo não somente uma maior importância ao trabalho feito por pessoas em profissões com maiores salários, como também atribuindo maiores níveis de competências aos profissionais que recebem níveis de remuneração consideráveis. Corroboram também aquelas investigações apontando que esta consciência das diferenças entre salários são muito mais marcantes nos participantes de NSE médio e alto do que nos participantes de NSE baixo.

A importância da experiência sociocultural na representação da desigualdade econômica torna-se particularmente evidente a partir da análise multidimensional, que para ser mais bem compreendida precisa de uma discussão mais aprofundada. As três comparações entre os perfis dos gru- 
pos investigados mostram haver uma estrutura modular subjacente à representação do nível de remuneração das ocupações em nossa sociedade. Como explicar esta estrutura modular? Qual o seu significado para uma compreensão dos níveis de representação dos grupos comparados?

Uma estrutura modular é caracterizada por aspectos específicos como: (a) uma determinada faceta apresentar, espacialmente muito próximos entre si, os pontos que a compõem; (b) a região formada por esta faceta encontrar-se numa região central da figura; (c) em volta desta região estarem dispostos os pontos de uma segunda ou terceira facetas. Compreender estas características a partir dos nossos dados nos possibilitará melhor compreender o significado subjacente desta estrutura e do nível de representação do objeto que a produziu.

A maior proximidade dos perfis dos participantes de NSE médio ao serem comparados com os outros dois grupos; NSE baixo e Meninos de rua indicam haver uma maior similaridade entre os perfis produzidos pelos membros deste grupo. Esta maior similaridade foi devida ao fato destes participantes possuírem um referencial similar para estabelecer os julgamentos das remunerações, sendo este referencial a realidade na qual vivem. Provavelmente, a conjunção do fato de utilizarem um mesmo referencial (os dados da realidade), possuírem maior conhecimento deste referencial, juntamente com o fato que nos julgamentos não se encontrarem contemplados dados relativos ao que eles desejariam que fosse, levou os participantes de NSE médio a uma maior similaridade em seus julgamentos e conseqüente mais proximidade espacial dos perfis nas projeções ao serem comparados com os participantes de NSE baixo e os Meninos de rua.

O fato dos participantes destes últimos dois grupos encontrarem-se espalhados em volta desta região central mostra que (a) estes participantes não compartilham, da mesma forma como os de NSE médio, a maneira como se encontram distribuídos em nossa sociedade os salários, (b) apesar da diversidade em seus julgamentos, estes últimos refletem, em última análise, a mesma realidade compartilhada pelos participantes de NSE médio. Portanto, a diferença que caracteriza os participantes de NSE baixo e Meninos de rua em relação aos participantes de NSE médio é a maneira como este conhecimento comum, a realidade, é compartilhado: de maneira muito mais fiel e próxima da realidade nos participantes de NSE médio e de maneira muito menos fiel e mais distante da realidade nos participantes de NSE baixo e Meninos de rua. A diferença que caracteriza estes dois últimos grupos em relação ao grupo de NSE médio, é a mesma que se observa também entre o grupo de participantes de NSE baixo em relação ao grupo Meninos de rua. O grupo de participantes de NSE baixo, ao ser comparado ao grupo dos Meninos de rua, apresenta uma maior similaridade em seus julgamentos sobre remuneração. Os motivos devem ser provavelmente os mesmos que expomos anteriormente em relação às outras duas comparações (NSE médio com NSE baixo e Meninos de rua).

Do ponto de vista teórico esta estrutura relacional modular pode ser representada da seguinte forma na Figura 9. Como é possível observarmos no modelo representacional que foi inferido, a partir dos dados, os julgamentos em termos de remuneração dos três grupos de participantes refletem o mesmo foco que é a realidade social. É a partir deste mesmo foco que os três grupos de participantes produzem seu nível específico de representação da realidade. Quanto maior o nível de conhecimento e menos contaminados por variáveis como o "nível de desejo", mais próximo o grupo se localizará em relação a este núcleo central, visto que este núcleo é compartilhado de forma mais similar. De fato, julgamentos que se fundamentam a partir do "nível de desejo" apresentam características da esfera pessoal e subjetiva, e consequentemente, sujeitas a maiores variações quando comparados com julgamentos que se fundamentam a partir de "dados de realidade" (mesmo considerando as suas limitações de subjetividade destas últimas).

Estes resultados são particularmente importantes devido às implicações na compreensão sobre o significado do valor da educação entre participantes pertencentes a diferentes NSE. Eles apontam na mesma direção daqueles apresentados em Roazzi et al. (1996) que visava compreender a transmissão intergeracional de status social e educacional. A perspectiva que se consolida a partir deste conjunto de investigações é que este fenômeno resulta de um processo de socialização que é simultaneamente cognitivo e social (para maiores detalhes ver Nunes, \& Roazzi, 1999). Neste sentido, crianças e adolescentes de NSE diferentes construiriam mundos consensuais desiguais oferecendo às crianças/adolescentes de NSE médio "razões para aprender" e às crianças/adolescentes de NSE baixo "razões para não aprender" na escola. Estas diferenças não decorreriam necessariamente de crenças gerais sobre o valor da educação, mas das representações específicas que as crianças e adolescentes possuiriam da estratificação social e educacional (Roazzi et al., 1996) e do nível de remuneração (a presente investigação), da identidade neste mundo social e dos tipos de conhecimentos, nos quais valeria a pena investir, em

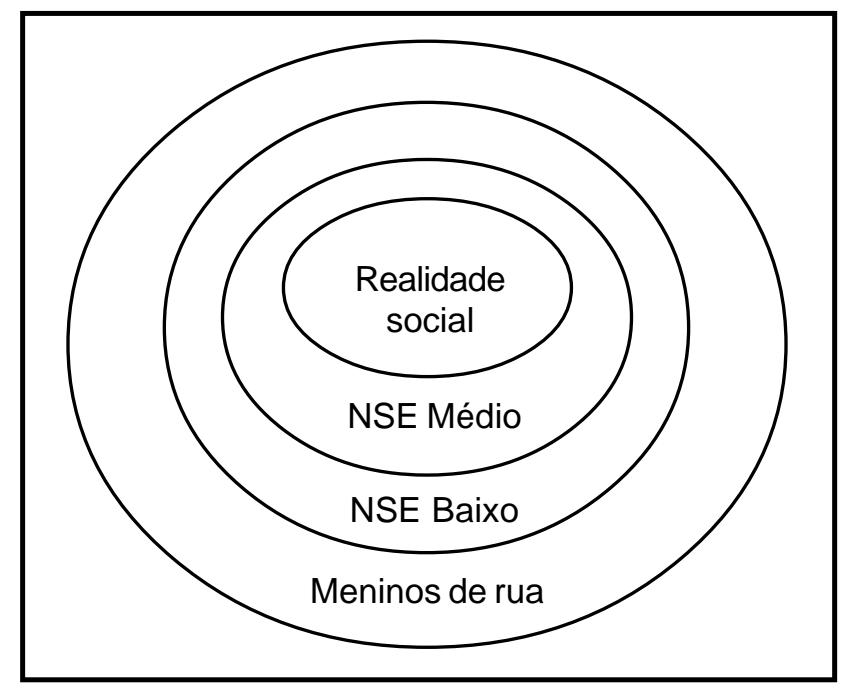

Figura 9. Representação modular da relação entre os diferentes grupos sendo comparados através das análises SSA. 
função da identidade e experiência sociocultural. Enfim, o conjunto destes resultados e considerações nos permitem compreender melhor as "dificuldades" apontadas por Willis (1977) ao explicar as diferenças que caracterizam as crianças de classes sociais diferentes em relação às escolhas profissionais quando afirma: "The difficult thing to explain about how middle class kids get middle class jobs is why others let them. The difficult thing to explain about how working class kids get working class jobs is why they let themselves" (p. 1, grifo nosso).

\section{Referências}

Barros, R. P., Henriques, R., \& Mendonça, R. (2000). Estabilidade inaceitável: Desigualdade e pobreza no Brasil. In R. Henriques (Ed.), Desigualdade e pobreza no Brasil (pp. 21-47). Rio de Janeiro, RJ: IPEA.

Borg, I., \& Groenen, P. J. F. (2005). Modern multidimensional scaling: Theory and applications. New York: Springer-Verlag.

Burgard, P., Cheyne, W. M., \& Jahoda, G. (1989). Children's representation of economic inequality: A replication. British Journal of Developmental Psychology, 7, 275-287.

Carraher, T. N., \& Schliemann, A. D. (1982). Fracasso escolar: Uma questão social. Mimeografado. Recife, PE: Universidade Federal de Pernambuco.

Chafel, J. A. (1996). Children's views of social inequality: A review of research and implications for teaching. The Educational Forum, 61, 46-57.

Danziger, S., \& Gottschalk, P. (1995). America unequal. Cambridge, MA: Harvard University Press.

Dickinson, J. (1990). Adolescent representations of socioeconomic status. British Journal of Developmental Psychology, 8, 351-371.

Duncan, G. J., \& Brooks-Gunn, J. (Eds.) (1997). Consequences of growing up poor. NewYork: Russel Sage.

Duncan, G. J., Brooks-Gunn, J., \& Klebanov, P.K. (1994). Economic deprivation and early childhood development. Child Development, 65,296-3 19.

Emler, N., \& Dickinson, J. (1985). Children's representations of economic inequality. British Journal of Developmental Psychology, 3, 191-198.

Furby, L. (1979). Inequalities in personal possessions: Explanations for and judgments about unequal distribution. Human Development, 22, 180-202.

Glyn, A., \& Miliband, D. (1994) Introduction. In A. Glyn \& D. Miliband (Eds.), Paying for inequality: The economic cost of social injustice (pp. 12-26). London: Rivers Oram Press.

Jahoda, G. (1983). European 'lag' in the development of an economic concept: A study in Zimbabwe. British Journal of Developmental Psychology, 1, 113-120.

Jones, R. M. (1995). The price of welfare dependency: Children pay. Social Work, 4O(4) 496-505.
Kerstenetzky, C. L. (2002). Por que se importar com a desigualdade. Dados, 45(4) 649-675.

Leahy, R. L. (1981). The development of the conception of economic inequality: I. Descriptions and comparisons of rich and poor people. Child Development, 52, 523-532.

Leahy, R. L. (1983). The development of the conception of economic inequality: II. Explanations, justifications, and concepts of social mobility and change. Developmental Psychology, 19(1), 111-125.

Leahy, R. L. (1990). The development of concepts of economic and social inequality. New Directions for Child Development, 46, 107-120.

Leiser, D. (1983). Children's conceptions of economics: The constitution of a cognitive domain. Journal of Economic Psychology, 4, 297-317.

Leiser, D., Sevón, G., \& Levy, D. (1990). Children's economic socialization: Summarizing the cross-cultural comparison of ten countries. Journal of Economic Psychology, 11, 591-614.

Nunes, T., \& Roazzi, A. (1999). Education, social identity, and occupational aspirations in Brazil: Reasons for (not) learning. In F. E. Leach \& A. W. Little (Eds.), Education, cultures and economics: Dilemmas for development (pp. 327-343). New York: Falmer Press.

Roazzi, A. (1988). Children's cognitive skills. Tese de Doutorado não-publicada, University of Oxford, UK.

Roazzi, A. (1995). Categorização, formação de conceitos e processos de construção de mundo: Procedimento de classificações múltiplas para o estudo de sistemas conceituais e sua forma de análise através de métodos de análise multidimensionais. $\mathrm{Ca}$ dernos de Psicologia, 1, 1-27.

Roazzi, A., Sales, L. C., \& Nunes, T. (1996, Agosto). Reason for (not) learning: The social representation of knowledge, economic inequality and social stratification. Trabalho apresentado no XIV Biennial International Society for the Study of Behavioural Development Conference, Québec City, Canadá.

Sklar, H. (1995). Chaos or community: Seeking solutions, not scapegoats for bad economics. Cambridge, MA: South End Press.

Weinger, S. (2000). Economic status: Middle class and poor children's views. Children \& Society, 14, 135-146.

Wilkinson, R. (1994a). Unfair shares: The effects of widening income differences on the welfare of the young. London: Barnardo's.

Wilkinson, R. (1994b). Health, redistribution and growth. In A. Glyn \& D. Milband (Eds.), The economic cost of social injustice (pp. 34-43). London: Rivers Oram Press.

Wilkinson, R. (1996). Unhealthy societies: The afflictions of inequality. New York: Routledge

Willis, P. (1977). Learning to labour. How working class kids get working class jobs. London: Saxon House.

Recebido: 19/10/2004

$1^{a}$ revisão: 01/06/2005

$2^{a}$ revisão: 16/08/2005

Aceite final: 23/08/2005 